Bio - grafía. Escritos sobre la Biología y su Enseñanza. ISSN 2027-1034

Edición Extraordinaria. p.p. 1060 - 1068

Memorias del IX Encuentro Nacional de Experiencias en Enseñanza de la Biología y la Educación Ambiental. IV Congreso Nacional de Investigación en Enseñanza de la Biología.

\title{
ENSEÑANZA DE LOS INSECTOS Y SUS INTERACCIONES ECOSISTÉMICAS CON ESTUDIANTES DEL GRADO SÉPTIMO DE LA INSTITUCIÓN EDUCATIVA EL PROGRESO FUENTEDEORO (META)
}

\author{
TEACHING OF THE INSECTS AND ITS ECOSYSTEM INTERACTIONS WITH \\ STUDENTS OF THE SEVENTH DEGREE OF THE INSTITUCIÓN EDUCATIVA EL \\ PROGRESO FUENTEDEORO (META)
}

Fernanda Fontecha Parra ${ }^{1}$

\section{RESUMEN:}

Esta investigación fue realizada en la Institución Educativa El Progreso (INEPRO) ubicada en el municipio de Fuentedeoro (Meta) como parte de la práctica pedagógica, que se llevó a cabo durante los meses de Julio a Noviembre del 2016 que consistió en la implementación de diferentes temáticas que se relacionan con el concepto de insectos dirigidas a los estudiantes del grado Séptimo (701-702) de la jornada de la mañana.

Este proyecto se realizó bajo un enfoque cualitativo relacionado con un paradigma interpretativo y tiene como objetivo aportar a los saberes de los estudiantes de la Institución desde la perspectiva de aprendizaje significativo, donde se tiene en cuenta los conocimientos previos sobre las interacciones que existen entre insectos y sus ecosistemas y como desde la enseñanza de estas problemáticas logren valorar su contexto rural. Con ayuda de un test se evidenciaron los conocimientos previos sobre las interacciones que establecen los estudiantes entre insectos y sus ecosistemas, en esta se obtuvo que existían falencias para describir e identificar insectos y su relación con el ecosistema; se realizan diferentes actividades de clase que contribuyen ampliar los saberes de los estudiantes teniendo en cuenta sus conocimientos previos. Como resultado se obtuvo que los estudiantes entendieron la importancia de los insectos a partir del conocimiento de sus interacciones que estos tienen con el ecosistema, donde también se obtuvo una guía ilustrada de los insectos que se encontraron en el colegio.

PALABRAS CLAVE: Insecto, Interacción Biológica, Ecosistema, Guía de Insectos, Ruralidad Educativa.

\section{ABSTRAC:}

This is a report of an investigation made during teaching practice carried out in the Educational Institution El Progreso (INEPRO) located in the municipality of Funtedeoro

${ }^{1}$ UPN 
Bio - grafía. Escritos sobre la Biología y su Enseñanza. ISSN 2027-1034

Edición Extraordinaria. p.p. 1060 - 1068

Memorias del IX Encuentro Nacional de Experiencias en Enseñanza de la Biología y la Educación Ambiental. IV Congreso Nacional de Investigación en Enseñanza de la Biología.

(Meta), around the research "science education in urban and rural contexts" within the Department Biology of the National Pedagogical University. a pedagogical strategy July 11 to November 11, 2016 which consisted of the implementation of different issues that relate to the concept of insect aimed at Seventh graders (701-702) of the day in the morning was developed. This project has a qualitative approach related to an interpretive paradigm. It aims to contribute to the knowledge of students of the institution from meaningful learning, which take into account prior knowledge about the interactions between insects and their ecosystems. This investigation began with a test of previous ideas that were conducted where students was evident that there were shortcomings to describe and identify insects and their relationship to the ecosystem; different classroom activities which contributed to the knowledge of students taking into account their prior knowledge were made. As a result, it was found that the students understood the importance of insects from the interactions they have with the ecosystem, where an illustrated guide insects were found in the school was also obtained.

KEYWORDS: Insect, Biological Interaction, Ecosystem, Insect Guide.

\section{INTRODUCCIÓN}

Ruralidad, es uno de temas centrales que maneja la línea de investigación "Enseñanza de la Biología en contextos rurales y urbanos" desde la cual se tiene como objeto visitar, conocer y fortalecer las dinámicas de ámbitos rurales y aportar de manera positiva en las mismas teniendo en cuenta aspectos biológicos, culturales y ancestrales de la región. Es por esta razón que como maestra en formación, se hace importante buscar alternativas para la enseñanza de la biología, que por medio de las interacciones que se promueve estudiar y observar en la clase (sujeto- medio, sujeto- insecto, sujeto -sujeto, insectomedio) con los jóvenes y con su entorno, se logra un aprendizaje desde la parte disciplinar, pedagógica e incluso en el proceso de crecimiento del sujeto. Comprendiendo además que en el contexto del Municipio de Fuentedeoro (Meta) hay una serie de problemáticas, conductas sociales y culturales que permiten el enriquecimiento de la práctica pedagógica, ya que estos espacios permiten conocer la vida rural, y cómo desde la pedagogía se puede aportar un aprendizaje a estos niños.

Las escuelas rurales en Colombia nos brindan espacios de enseñanza diferentes, donde podemos demostrar que como licenciados en Biología podemos salir de la cotidianidad y monotonía de las clases, para realizar actividades que interesen a los estudiantes por lo vivo y la vida, donde la enseñanza-aprendizaje se vea motivada a los estudiantes a comprender la vida y lo vivo. La biología por tratarse de una ciencia que estudia la vida, no debe centrarse en una educación donde solo preste importancia el conocimiento científico, ya que esta suele ser diversa y por esto las maneras de comprenderlas siempre serán diferentes, y aún más en zonas rurales donde se puede ver con más claridad esos 
Bio - grafía. Escritos sobre la Biología y su Enseñanza. ISSN 2027-1034

Edición Extraordinaria. p.p. 1060 - 1068

Memorias del IX Encuentro Nacional de Experiencias en Enseñanza de la Biología y la Educación Ambiental. IV Congreso Nacional de Investigación en Enseñanza de la Biología.

factores (interacciones entre los organismos con su territorio) ya que se tiene la naturaleza más cerca y esto facilita su observación.

Es por ello que el estudiante se debe encontrar en un ambiente escolar ameno, lo que significa que descubra un entorno más agradable y tranquilo, donde el aprendizaje no resulte una obligación, y no solo se limite a lo mismo que, aunque sean conceptos importantes de aprender no tiene valor intrínseco alguno si no hay apropiación de estos. Además, no debemos limitarnos a enseñar lo disciplinar, debemos formar personas para el futuro donde el estudiante se apropie como sujeto y aprenda a valorar su contexto.

Este proyecto tuvo como objetivo motivar a los estudiantes del Municipio, a participar como agentes educativos en el conocimiento de los organismos que allí habitan, comprendiendo la importancia de la biodiversidad que existe allí, Los insectos no son una herramienta en sí, el maestro es el que logra que la observación y el conocimiento de los mismos sea una experiencia didáctica, y que al reconocer se apropien del recurso biológico que poseen y así logren aportar a su conservación y a su vez podrán reconocer las diferentes interacciones biológicas entendidas como:

"Acción social que una especie lleva a cabo con otra u otras especies. Permite un entramado de relaciones sociales entre especies dentro de una comunidad en un ecosistema. Ningún organismo que viva en comunidad, ya sea un fragmento de matorral, un prado, un estanque o un arrecife de coral, existe de forma aislada. Cada organismo interviene en toda una serie de interacciones, tanto con otros organismos como con los factores del ambiente abiótico" (Raven, Evert \& Eichhorn 1992)

Que tienen los organismos con la naturaleza y cómo estas ayudan a que los ecosistemas mantengan una homeostasis.

Es por esto que desde la enseñanza de los insectos y a partir de las prácticas convivenciales y de enseñanza biológica, se busca que los estudiantes logren comprender la importancia y el reconocimiento del contexto rural al cual pertenecen, así como reconocer los saberes de los estudiantes y desde allí ampliar los conocimientos, y con esto crear un ambiente escolar propicio para la enseñanza de esta, donde es clave buscar diferentes alternativas de aprendizaje ya que es una base importante en esta sociedad para lograr integrar todos los procesos pedagógicos enseñados en la formación docente.

La finalidad de este proyecto es lograr que los estudiantes de la Institución Educativa El Progreso (INEPRO) apropien su conocimiento a partir de las interacciones entre los insectos y el ecosistema visto como:

"Conjunto de especies de un área determinada que interactúan entre ellas y con su ambiente abiótico; mediante procesos como la depredación, el parasitismo, la competencia y la simbiosis, y con su ambiente al desintegrarse y volver a ser parte del ciclo de energía y de nutrientes. Las especies del ecosistema, incluyen bacterias, hongos, plantas y animales dependen unas de otras. Las relaciones 
Bio - grafía. Escritos sobre la Biología y su Enseñanza. ISSN 2027-1034

\section{Edición Extraordinaria. p.p. 1060 - 1068}

Memorias del IX Encuentro Nacional de Experiencias en Enseñanza de la Biología y la Educación Ambiental. IV Congreso Nacional de Investigación en Enseñanza de la Biología.

entre las especies y su medio, resultan en el flujo de materia y energía". (Glowka, 1996)

y como desde la enseñanza de estas logren valorar su contexto rural.

\section{METODOLOGÍA}

Este proyecto tiene una metodología cualitativa y es de un paradigma interpretativo, porque permite comprender e interpretar la realidad, los significados de los estudiantes, percepciones, intenciones, acciones, respecto a las interacciones con los insectos; desde este paradigma se ve la relación sujeto/objeto como dependiente lo que haga el uno afecta al otro; los valores están presentes en la investigación e influyen en la selección del problema, teoría, método y análisis (Quiroga, 2007). El trabajo de investigación se dividió en tres fases, la primera fase de diagnóstico, la segunda de la realización de clases con el taller final y la tercera fase de sistematización y análisis de resultados.

Para este trabajo se desarrollaron estrategias didácticas que incluían test ideas previas, clases magistrales y realización de Guía llustrada de Insectos del colegio, que se implementaron desde el 11 de Julio y finalizaron el 11 de noviembre del 2016, con los estudiantes del grado Séptimo (701-702) de la jornada mañana de la IE El Progreso (INEPRO). El Test de ideas previas el cual consistía en saber que conocían los estudiantes acerca de los insectos que ellos habían visto en el colegio; las clases tenían como el fin de aportar el fundamento teórico-práctico para de esta forma dar inicio a la construcción de la guía de insectos. Por último, el taller final consistió en la realización de una actividad lúdica donde los estudiantes debían representar mediante una dramatización las interacciones que un insecto presenta con su ecosistema, y de esta manera evaluar cualitativamente los conocimientos adquiridos por los estudiantes durante la investigación.

\section{RESULTADOS}

En la fase diagnóstico, se conocieron las ideas previas que tenían los estudiantes sobre los insectos, para ello, se formularon preguntas como. Las preguntas base fueron: ¿qué es un insecto?, tipos de insectos que conozca, menciona 10, Dibuja un insecto con sus partes, ¿Qué tipo de locomoción presentan los insectos?, ¿En qué hábitat se encuentran los insectos? Donde los resultados fueron 
Bio - grafía. Escritos sobre la Biología y su Enseñanza. ISSN 2027-1034

Edición Extraordinaria. p.p. 1060 - 1068

Memorias del IX Encuentro Nacional de Experiencias en Enseñanza de la Biología y la Educación Ambiental. IV Congreso Nacional de Investigación en Enseñanza de la Biología.

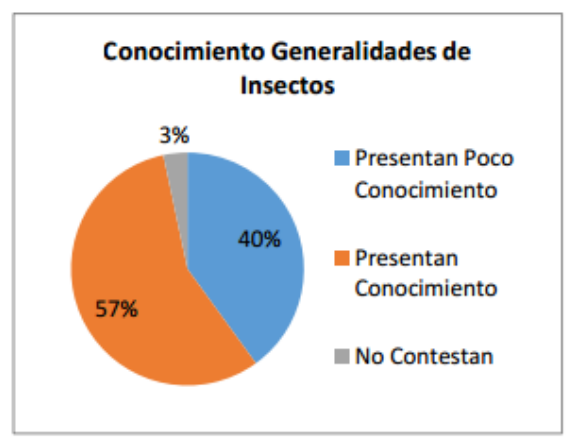

Gráfico 1: Diagnostico Ideas Previas. Muestra los porcentajes de los estudiantes del grado séptimo (701-702) IE El Progreso, acerca de los conocimientos previos de generalidades de insectos.

El gráfico 1 indica que un alto porcentaje de los estudiantes tiene conocimiento sobre las generalidades de los insectos, afirmando que un insecto es un animal pequeño e invertebrado que presenta quitina, que tienen distintos tipos de locomoción y se encuentran en distintos hábitats. Las principales falencias se centran en confundir ciertos organismos como araña, lombriz, salamanqueja, escorpión, lagartija, babosa, rana, garrapata, colorado, introduciéndolos en el grupo de los insectos. Ver gráfico 2.

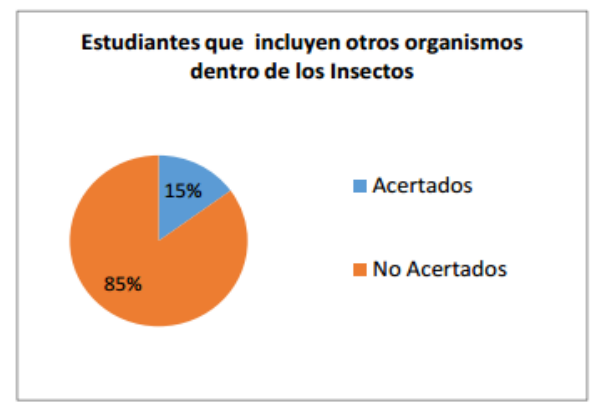

GRÁFICO 2: Porcentaje de estudiantes que confundieron otro grupo de organismos con los insectos. Estudiantes de grado Séptimo que incluyen organismos diferentes.

En la segunda fase, se desarrollaron clases que tenían como fin dar el fundamento teórico-práctico para de esta forma dar inicio a la guía de insectos. Lo que se pretendía con esto era que a medida que se realizaban las clases los estudiantes empezaran a elaborar la guía, con el objetivo de desarrollar habilidades de identificar insectos a nivel de orden a partir del dibujo. 
Bio - grafía. Escritos sobre la Biología y su Enseñanza. ISSN 2027-1034

Edición Extraordinaria. p.p. 1060 - 1068

Memorias del IX Encuentro Nacional de Experiencias en Enseñanza de la Biología y la Educación Ambiental. IV Congreso Nacional de Investigación en Enseñanza de la Biología.

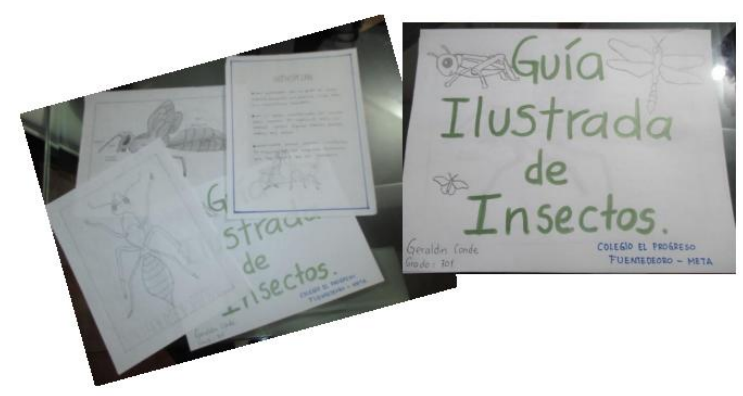

Imagen 1: Guía llustrada de Estudiante INEPRO.

Los estudiantes se mostraban muy interesados al momento de realizar la actividad, ya que la mayoría muestra gran habilidad en el dibujo y esto facilito que diferenciaran los distintos órdenes.

Esta fase se desarrolló durante 8 sesiones, en las primeras seis a los estudiantes se les proyectaba imágenes de cuatro órdenes de insectos por sesión, donde se les hablaba de las generalidades de cada uno; en las dos últimas sesiones los estudiantes reconocieron los órdenes que habían visto en el colegio y así pudieron hacer una pequeña colecta de ellos e identificación la cual registraron en la guía.

La última parte de esta fase, fue un taller final el cual consistió en la realización de una actividad lúdica donde los estudiantes debían completar una frase sobre las interacciones que los insectos presentan con el ecosistema y dar un ejemplo. Se hicieron grupos donde a cada grupo se le hacia una pregunta respecto a las interacciones biológicas, y el grupo que contestaba correctamente se gana una parte de la frase que tenían que completar.

Con lo anterior, se pudo evidenciar que en su mayoría los estudiantes lograron entender el tema de las interacciones biológicas ya que erraron en pocas preguntas, así como lo muestra la gráfica 3 . 
Bio - grafía. Escritos sobre la Biología y su Enseñanza. ISSN 2027-1034

\section{Edición Extraordinaria. p.p. 1060 - 1068}

Memorias del IX Encuentro Nacional de Experiencias en Enseñanza de la Biología y la Educación Ambiental. IV Congreso Nacional de Investigación en Enseñanza de la Biología.

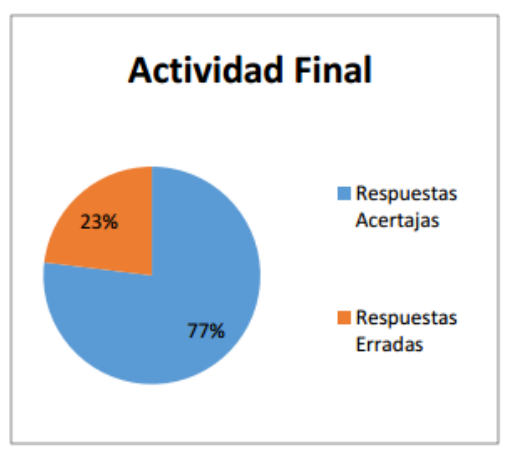

GRÁFICA 3: Resultados Actividad Final. Muestra el porcentaje de preguntas acertadas contra preguntas erradas de los estudiantes de grado séptimo.

\section{DISCUSIÓN}

Entrarse en nuevos escenarios para la enseñanza de la biología significa un gran reto para los maestros en formación, puesto que el desarrollo de estrategias alternativas de educación en las instituciones educativas no siempre es bien recibida.

La implementación de la guía de insectos como estrategia con los estudiantes del grado séptimo (701-702) de la Institución Educativa El Progreso (INEPRO) mostro resultados que son importantes tener en cuenta a la hora de la implementación de la práctica pedagógica, puesto que nos da una idea de cómo están viendo los estudiantes la enseñanza de la biología y como ellos mismos pueden cambiar la forma de enseñar y aprender de esta.

Al enseñarse las interacciones que tienen los insectos con el ecosistema resulta mucho más fácil abordarla desde las zonas verdes que se encuentran en las instalaciones de la institución, ya que aunque el libro de texto da un fundamento teórico que es importante no es igual que presenciar estas interacciones de forma natural.

Temas como las redes tróficas e interacciones ecológicas, resultaron fácil de abordar a partir de la observación en campo, donde los estudiantes reconocieron e identificaron ordenes vistos en el salón de clase, estos ordenes incluían Odonata, Orthoptera, Hemípteros, Isóptera, Blattodea, Lepidóptera, Díptera, Coleóptera e Hymenoptera, donde se pudo evidenciar relaciones interespecíficas como depredación, competencia, cooperación y relaciones intraespecíficas como la cooperación.

La principal falencia que se vio fue la función de los estudiantes en el momento de la identificación, puesto que el $85 \%$ de ellos confundían los insectos con otros organismos. Esto da a entender que existe una falencia en el área de ciencias Naturales frente al reconocimiento de los organismos más cercanos a nuestro entorno. Al utilizar la Guía de 
Bio - grafía. Escritos sobre la Biología y su Enseñanza. ISSN 2027-1034

Edición Extraordinaria. p.p. 1060 - 1068

Memorias del IX Encuentro Nacional de Experiencias en Enseñanza de la Biología y la Educación Ambiental. IV Congreso Nacional de Investigación en Enseñanza de la Biología.

Insectos como estrategia didáctica resulto una experiencia nueva para ellos, porque estaban acostumbrados a la clase de tablero donde se limitan a copiar y memorizar según los estudiantes, además se potencio habilidades tales como el dibujo y mostro a los estudiantes que se puede aprender al tiempo que se viven experiencias diferentes dentro del salón de clase.

\section{CONCLUSIONES}

- Los estudiantes al conocer la biota que los rodea le dan un valor intrínseco lo que permite que apropien su entorno rural

- La implementación de estrategias diferentes como la recolección de insectos en la institución permiten a los estudiantes establecer relaciones entre los contenidos teóricos de las clases con las prácticas llevadas a cabo en espacios diferentes al aula como lo son las zonas verdes.

- Al realizar actividades lúdicas los estudiantes muestran mejor entendimiento y comprensión, y por ende muestran un mayor interés.

- Al llevar a cabo la Guía de Insectos se logró reforzar habilidades como el dibujo que son poco utilizadas en el salón clase, que aunque son poco implementadas son muy importantes para el desarrollo de otras formas de inteligencias.

\section{REFERENTES}

- Angulo, G. (2015) Minería en el Meta. Análisis de una minería sin oriente.

- Ausubel, D. P. (1976). Psicología educativa: Un punto de vista cognoscitivo. Mexico: Trillas.

- Ausubel, D. P. (2002). Adquisición y retención del conocimiento: una perspectiva cognitiva. Barcelona, España: Paidós lbérica, S.A.

- Cusicanqui, J. M. (2008). EL ARTE DE APRENDER... Y DE ENSEÑAR; Manual para docentes. Santa cruz de la sierra, Bolivia: La Hoguera.

- Eichhorn, R., Evert, H., Raven, P. (1992) Biología de las Plantas. Editorial Reverté S.A. Barcelo, Bogotá, Buenos Aires, México. 
Bio - grafía. Escritos sobre la Biología y su Enseñanza. ISSN 2027-1034

Edición Extraordinaria. p.p. 1060 - 1068

Memorias del IX Encuentro Nacional de Experiencias en Enseñanza de la Biología y la Educación Ambiental. IV Congreso Nacional de Investigación en Enseñanza de la Biología.

- Glowka, L., Burhenne, F. \& Synge, H. (1996) Guía del convenio sobre la Diversidad Biológica. UICN.

- Guber, Rosana.(2001) La etnografía: método, campo y reflexividad. Editorial Norma.

- Kemmis, s. \& Carr, W. (1988). Teoria critica de la enseñanza, la investigación-accion en la formación del profesorado. España: Editores Martinez Roca.

- Méndez, Z. (2001) Aprendizaje y Cognición. Editorial Universidad Estatal a Distancia

- PRAE (2016) Institución Educativa El Progreso. 